\title{
An overview of breast cancer epidemiology, risk factors, pathophysiology, and cancer risks reduction
}

\begin{abstract}
Cancer results from a series of molecular events that fundamentally alter the normal properties of cells. They are abnormal cells in which the processes regulating normal cell division are disrupted. There are several types of cancer. Breast cancer is a disease in which malignant (cancer) cells form in the tissues of the breast. Breast cancer is the most common female cancer, the second most common cause of cancer death in women. Over a million new cases of breast cancer are diagnosed every year. Both mortality and burden are high. Due to lack of information, basic knowledge on cancer, poor health facilities, and poor access to treatment, late screening and detection, breast cancer mortality is higher in developing nations, even though incidence rates are higher in developed nations. To increase breast cancer awareness this paper presents a review on breast cancer epidemiology, classification, the pathology of breast cancer, risk factors, and ways of preventing breast cancer through risk factors reduction.
\end{abstract}

Keywords: breast cancer burden, etiology, risk factors, malignant, invasive carcinoma, disease, tumour, BRCA-1, BRCA-2
Volume I Issue 4 - 2017

Lord Tertese Angahar

Department of Biological Sciences, Federal University of Agriculture, Nigeria

Correspondence: Lord Tertese Angahar, Department of Biological Sciences, Federal University of Agriculture, P.M.B 2373 Makurdi, Nigeria, Email lordlegs365@gmail.com

Received: June 21, 2017 | Published: July 10, 2017

\section{Introduction}

Cancer is a world-wide public health problem affecting all categories of persons. It is among the major global health issues, with an estimated 10 million incidences and 6 million annual mortality rates. ${ }^{1}$ Boyle et al. ${ }^{2}$ reported that, by $2030,70 \%$ of all new cases will be found in developing countries. Ferlay et al., ${ }^{3}$ observed that in 2008, there were 12.7 million cases and 7.6 million cancer mortality with Nigeria accounting for more than 10,000 cancer deaths and about 250,000 incidences are recorded annually. Cancer results from a series of molecular events that fundamentally alter the normal properties of cells. The normal control systems that prevent cell overgrowth and the invasion of other tissues are disabled in cancer cells. ${ }^{4}$ In other words, Cancer is a disease which occurs when changes in a group of normal cells within the body leads to uncontrolled growth causing a lump called a tumour; this is true of all cancers except leukaemia (cancer of the blood). If left untreated, tumours can grow and spread into the surrounding normal tissue, or to other parts of the body via the bloodstream and lymphatic systems, and can affect the digestive, nervous and circulatory systems. ${ }^{5}$ All cancer cells are abnormal cells in which the processes regulating normal cell division are disrupted. The incidence and burden of cancer is huge and is set to rise. Cancer kills more people on a global scale than AIDS, malaria and TB combined. ${ }^{6}$ It has been observed that the incidence of cancer is highest in developed countries, particularly in Northern America, Australia and New Zealand and in Northern and Western Europe. However, its impact in the developing world is fast growing. More than $70 \%$ of all cancer deaths already occur in low- and middle-income countries and these regions are projected to account for two-thirds of all cases of cancer worldwide by $2050{ }^{6}$

It was reported that about 24.6 million people are living with cancer worldwide. ${ }^{7} 12.5 \%$ of all deaths are attributable to cancer and if the trend continues, it is estimated that by 2020, 16 million new cases will be diagnosed per annum out of which $70 \%$ will be in developing countries. ${ }^{7}$ The exact burden of cancer in Nigeria and many African countries is not known because of lack of statistics, under-reporting, and inaccurate population statistics which makes age specific incidence rates impossible or if available inaccurate. ${ }^{7}$ Also, many patients who patronize orthodox medicine are not recorded. In a study of cancer registry literature update from all over the world, only $1 \%$ of the literature came from Africa as compared to $34 \%$ and $42 \%$ from Europe and Asia respectively. Although statistics has shown higher incidence and prevalence of cancer in the developed than the developing world, cancer burden and mortality are higher in developing nations. ${ }^{89}$ This is due to lack of information, basic knowledge on cancer, poor health facilities, and poor access to treatment, late screening and detection. The objectives of this review is to add to the body of knowledge, information on the epidemiology of breast cancer, risk factors, breast cancer pathology, risk factors reduction and prevention, with the aim of reducing breast cancer incidence, prevalence and burden.

\section{Classification of cancer}

Cancer can be classified according to the following categories: ${ }^{10}$ Carcinoma-This cancer arises from the epithelial cells (the lining of cells that helps protect or enclose organs). Carcinomas may invade the surrounding tissues and organs and metastasise to the lymph nodes and other areas of the body. The most common forms of cancer in this group include: breast, prostate, lung and colon cancer. SarcomaThis is a type of malignant tumour of the bone or soft tissue (fat, muscle, blood vessels, nerves and other connective tissues that support and surround organs). The most common forms of sarcoma are leiomyosarcoma, liposarcoma and osteosarcoma. ${ }^{11}$ Lymphoma is a cancer of the lymphatic system, which runs all through the body, and can therefore occur anywhere. The two main forms are non-Hodgkin's which begins with uncontrolled growth of the - white blood cells -lymphocytes - of the immune system. ${ }^{11}$ and Hodgkin's lymphoma in which cells of the lymph nodes become cancerous. Leukemia is a cancer of the white blood cells and bone marrow, the tissue that forms blood cells. There are several subtypes; common are lymphocytic leukaemia and chronic lymphocytic leukaemia. ${ }^{11,12}$ The abnormalities in cancer cells usually result from mutations in protein-encoding genes 
that regulate cell division. Over time more genes become mutated. This is often because the genes that make the proteins that normally repair DNA damage are themselves not functioning normally because they are also mutated. Consequently, mutations begin to increase in the cell, causing further abnormalities in that cell and the daughter cells. Some of these mutated cells die, but other alterations may give the abnormal cell a selective advantage that allows it to multiply much more rapidly than the normal cells. This enhanced growth describes most cancer cells, which have gained functions repressed in the normal, healthy cells. As long as these cells remain in their original location, they are considered benign; if they become invasive, they are considered malignant. Cancer cells in malignant tumors can often metastasize, sending cancer cells to distant sites in the body where new tumors may form. ${ }^{11,12}$

\section{Breast cancer}

The term breast cancer is an umbrella term for several sub-types of cancers of the breast. These breast cancer subtypes differs in their clinical presentation, reveal distinct gene expression patterns, and have different genetic and molecular characteristics. ${ }^{13,14}$ Breast cancer is a disease in which malignant (cancer) cells form in the tissues of the breast. It occurs in both sex, but very rare in men. Breast cancer is the most common cause of cancer death among women in 140 of 184 countries worldwide and the most frequently diagnosed cancer among women which now represents one in four of all cancers in women.

Table I The prevalence rates of cancer among female.
Breast cancer Epidemiology and BurdenBreast cancer is the most common female cancer, the second most common cause of cancer death in women, and the main cause of death in women ages 40 to $59 .{ }^{15}$ Breast cancer is a common form of cancer among women globally. Currently, it is the most leading cause of cancer death with 198,000 deaths per annum which represents $15.4 \%$ of all deaths in developed regions after that of the lung cancer. ${ }^{16}$ In developing countries, it is the first leading cause of death among women with 324,000 deaths which represented $14.3 \%$ of all deaths. ${ }^{16-18}$ It is second only to lung cancer as a cause of cancer mortality, and it is the leading cause of death for American women between the ages of 40 and $55 .{ }^{19}$ The lifetime risk of a woman developing invasive breast cancer is $12.6 \%$. Two one out of eight females in the United States will develop breast cancer at some point in her life. ${ }^{20}$ The Cancer Statistics Worldwide, ${ }^{21}$ documented that worldwide; more than one million new cases of female breast cancer are diagnosed each year, making it the most commonly occurring disease in women, accounting for over $1 / 3$ of the estimated annual 4.7million cancer diagnosis in females and the second most common tumor after lung cancer in both sexes. It is also the most common female cancer in both developed and developing countries with 55\% of it occurring in the developing countries. ${ }^{22}$ Garcia, ${ }^{23}$ reported that over one million women are diagnosed with breast cancer every year, and it is the leading cause of cancer death in women. Every year more than 500,000 women die from the disease. ${ }^{24}$ The prevalence of cancer among female in some African countries is presented in (Table 1) Adapted from Baba et al. ${ }^{25}$

\begin{tabular}{|c|c|c|c|c|c|c|c|}
\hline Cancer site & ICD & Benin\% & Cameroun\% & Chad\% & Niger\% & Nigeria\% & Total\% \\
\hline Bladder & $\mathrm{C} 67$ & 1.24 & 0.5 & 1.09 & 2.08 & 0.73 & 1.03 \\
\hline Brest & $\mathrm{C} 50$ & 34 & 35 & 36 & 41.5 & 41.2 & 39.9 \\
\hline Cervix uteri & C53 & 31 & 31 & 22.9 & 14.9 & 26.3 & 26.2 \\
\hline Colorectum & $C|8-2|$ & 4.16 & 3.4 & 4.62 & 7.97 & 3.63 & 4.45 \\
\hline Corpus uteri & $\mathrm{C} 54$ & 3.82 & 2.9 & 3.65 & 5.37 & 3.08 & 3.6 \\
\hline Kaposi sarcoma & $\mathrm{C} 46$ & 0.22 & 0.9 & 0.61 & 0.35 & 0.73 & 0.6 \\
\hline Larynx & $\mathrm{C} 32$ & 0.22 & 0 & 0 & 0.17 & 0.9 & 0.09 \\
\hline Leukaemia & $\mathrm{Cl} 19-95$ & 1.22 & 1.3 & 1.95 & 1.39 & 1.27 & 1.4 \\
\hline Lip, oral cavity & $\mathrm{C} 00-08$ & 2.36 & 2.4 & 2.19 & 2.77 & 1.09 & 2.07 \\
\hline Liver & $\mathrm{C} 22$ & 10 & 22.6 & 4.99 & 5.37 & 7.34 & 6.13 \\
\hline Lung & C $33-34$ & 1.01 & I & 1.09 & 0 & I & 0.9 \\
\hline Nasopharynx & $\mathrm{CII}$ & 0.22 & 1.6 & 1.09 & 0.17 & 0.54 & 0.76 \\
\hline Non-hodgkin lymp & C82-85, C96 & 2.47 & 8.3 & 4.99 & 2.25 & 2.54 & 4.22 \\
\hline Ovary & $\mathrm{C} 56$ & 3.6 & 5.1 & 6.57 & 12.7 & 2.81 & 5.49 \\
\hline Pancrease & $\mathrm{C} 25$ & 0.45 & 0.3 & 0.85 & 0.69 & 1.36 & 0.76 \\
\hline Stomach & $\mathrm{Cl} 6$ & 4.05 & 2.1 & 1.95 & 2.6 & 1.81 & 2.46 \\
\hline
\end{tabular}

\section{Development of breast cancer}

\section{Etiology of breast cancer}

The etiology of breast cancer is not fully understood. ${ }^{8}$ A variety of interrelated factors, such as genetics, hormones, the environment, sociobiology and physiology among others can influence breast cancer development.

\section{Risk factors for breast cancer}

Studies has shown that a number of conditions known as risk factors encourage or predisposes one to cancer. Some identified risk factors for breast cancer include:

i. Environmental factors: Exposure to ionizing radiation due to nuclear war, use of gadgets, medical diagnostic or therapeutic procedures increased risk of developing breast cancer. ${ }^{26}$ 
ii. Sociobiological factors: Gender and age are important risk factors for breast cancer development. Globally, $75 \%$ of new cases and $84 \%$ of breast cancer deaths occurs in women aged 50 and older, with the number of breast cancers diagnosed in women in their fourth decade of life rating at 1 in 232 compared to those in their seventh decade of life, which are rated at 1 in 29 . This increase may be directly related to hormonal changes in women in this age group. ${ }^{27,28}$

iii. Nutritional factors: High intake of fats increases the risk of developing breast cancer. Diet with high amounts of fat, caffeine and red meat is a positive risk factor for breast cancer. While the consumption of fruits and vegetables may reduce the risk of breast cancer development. ${ }^{28}$ Phytoestrogens and high amounts of calcium/vitamin D can also be effective to reduce breast cancer risks.

iv. Physiological factors: Moderate physical activities or exercise lowers the risks of breast cancer. Studies have shown a 30\% reduction in risk level associated with a few hours per week of vigorous activity compared to no exercise at all. ${ }^{28}$

v. Genetic factor: Although only $5 \%$ to $6 \%$ of breast cancers are considered hereditary, ${ }^{29}$ genetics plays a limited but important role as a risk factor for breast cancer.BRCA-1 and BRCA-2 account for an estimated $80 \%$ of hereditary breast cancer. BRCA-1 and/or BRCA-2 positive women have a $50 \%$ to $85 \%$ lifetime risk of developing breast cancer and a $15 \%$ to $65 \%$ risk of developing ovarian. ${ }^{30}$

vi. Family risk factors: Breast cancer is considered a risk if you have a family member who developed cancer. ${ }^{31}$ A cancer positive family history is a risk factor.

vii. Alcohol: The evidence that all types of alcoholic drinks are a cause of a number of cancers is now stronger than ever before. Alcohol can increase the risk of breast cancer, including mouth, throat, pharyngeal cancer, laryngeal, cancer of the foodpipe, liver, and bowel cancer (in men). Even moderate alcohol intake increases the risk of cancer. ${ }^{32}$

viii. A woman's hormonal history: The more menstrual cycles a woman has over her lifetime, the greater is her risk of breast cancer. ${ }^{33}$ The hormonal history of appears to be a risk factor, as the relative risk of breast cancer seems to be related to the breast's cumulative exposure to estrogen and progesterone. Early menarche (onset of menstruation, age 13), having no children or having them after age 30 , and menopause after age 50 and especially age 55 -all these mean more menstrual cycles and thus greater hormone exposure. ${ }^{34}$

ix. History of breast cancer: Women who were treated of breast cancer have the risk of developing a new second cancer in either the treated breast or the other breast. ${ }^{35}$

x. Obesity: Women who are obese have higher blood levels of the hormone oestrogen. This is because fat cells make oestrogen, which fuels the growth of most breast cancer tumours. ${ }^{33}$

xi. Hormone replacement therapy and oral contraceptives: Hormone replacement therapy and oral contraceptives are sources of oestrogen, which is a risk factor for breast cancer. ${ }^{33}$

xii. The immune system: People who have weakened immune systems are more at risk of developing some types of cancer. This includes people who have had organ transplants and take drugs to suppress their immune systems to stop organ rejection, plus people who have HIV or AIDS, or other medical conditions which reduce their immunity to disease. ${ }^{26}$

xiii. Tobacco: Over 80 different cancer-causing substances (carcinogenic agents) are present in tobacco smoke. When smoke is inhaled the chemicals enter the lungs, pass into the blood stream and are transported throughout the body. ${ }^{32}$ Smoking is thus an important risk factor for breast, lungs and other kinds of cancer.

xiv. Exposure to Cancer-causing substances (carcinogens): Exposure to cancer causing substances may cause mutation of normal cells to cancerous cells.

xv. Infections: Breast cancer may develop as a result of viral infections. Examples include cervical cancer, linked to the Human Papilloma Virus is linked to cervical cancer, while primary liver cancer is linked to the Hepatitis $\mathrm{B}$ and $\mathrm{C}$ virus. Lymphoma is linked to the Epstein-Barr virus. ${ }^{26,32}$

xvi. Pathology of the breast disease: Clinically, breast disorders can be described as follows:

xvii. Inflammatory lesions: These are rare breast lesions that can be acute or chronic and include acute mastitis, duct ectasia, posttraumatic lesions and granulomatous mastitis.

xviii. Benign fibrocystic lesions: Fibrocystic changes represent the single most common disorder of the breast and account for more than $40 \%$ of all surgical operations on the female breast. ${ }^{24}$ It is diagnosed frequently between the ages of 20 and 40 years, and rarely develops after menopause. It is frequently influenced by hormonal imbalance.

xix. Benign breast diseases: These are rare tumours, which include fibro adenomas, phyllodes tumours and large duct papilloma.

xx. Proliferative breast disorder: Epidemiological studies have identified changes in the breast resulting in an increased risk of developing carcinoma. This risk is due to hyperplasia with or without atypia. These lesions are often accompanied by fibrocystic changes as well. They can be associated with mammographic abnormalities. ${ }^{28}$

xxi. Carcinoma of the breast: Breast cancer can be divided into two main groups: non-invasive or carcinoma in situ, and invasive carcinoma. ${ }^{4}$

xxii. Common symptoms of Breast cancer: Symptoms of breast cancer vary from person to person. Some common breast cancer signs and symptoms include: Skin changes, such as swelling, redness, or other visible differences in one or both breasts, increase in size or change in shape of the breast(s). Changes in the appearance of one or both nipples, Nipple discharge other than breast milk, General pain in and or, on any part of the breast, Lumps or nodes felt on or inside of the breast; cancerous lumps are often painless and may increase in size as the cancer progresses. Unexplained weight loss over a short period of time (a couple of months) can be a sign of cancer. Extreme tiredness (fatigue) and a severe lack of energy. ${ }^{36}$

\section{Invasive breast cancer symptoms}

Symptoms more specific to invasive breast cancer include: Irritated or itchy breasts, Change in breast color, Increase in breast size or shape (over a short period of time), Changes in touch (may feel 
hard, tender or warm) Peeling or flaking of the nipple skin, A breast lump or thickening, Redness or pitting of the breast skin (like the skin of an orange). ${ }^{37}$

\section{Reducing your risk of breast cancer}

Although the presence of a number of risk factors is not a conclusion to the establishment of cancer, modifying the flexible risk factors has significance health benefits. One of the best ways to fight cancer is to prevent it from occurring, by identifying and controlling factors that increase a person's risk of developing the disease. We can control or reduce our exposures to ionizing radiation. Increase intake of vegetables, fruits, especially vegetables knows to be rich in important antioxidants and phytochemicals. Engaging in moderate physical activities, or exercise. Lack of physical activity is an established risk factor for premenopausal breast cancer and represents part of a complete approach to weight management. ${ }^{26}$ In addition, women who stay active can also reduce their risk of other diseases, such as coronary heart disease and colon cancer, and they can increase their quality of life. Of all the established risk factors for breast cancer, obesity and lack of physical activity stand out as the two that can be most readily and safely modified..$^{38}$ Avoid alcohol intake and tobacco. Control indiscriminate use or prolong use of contraceptive. Vaccination or prevention against viral infections such as Hepatitis A and C.

\section{Treatments of breast cancer}

Treatment of breast cancer may involve a number of approaches which are largely a function of several factors such as the stage of the disease, the state of the patient, among others. Treatment may involve: Surgery-Depending on the staging and type of the cancer, your doctor will discuss with you the most appropriate type of surgery, ranging from removal of the lump (lumpectomy) to removal of the whole breast (mastectomy) and surrounding tissues. Radiation therapy-It is a cancer treatment that uses high energy X-ray or other types of radiation to kill cancer cells or keep them from growing. Chemotherapy - It is a cancer treatment that uses drugs to stop the growth of cancer cells, either by killing the cells or by stopping them from dividing. Hormonal therapy-It is a treatment that removes hormones or blocks their action and stops cancer cells from growing. Targeted therapy-It is a type of treatment that uses drugs or other substances to identify and attack specific cancer cells without harming normal cells. ${ }^{39}$

\section{Conclusion}

Early detection of breast cancer can play a significant role in reducing its incidence and burden. Breast cancer mortality can be reduced if cases were detected and treated early. Constant mammograms and breast examinations nevertheless do not prevent cancer, but these screening tests enables it's early detection; when treatment is most likely to be effective. Although the presence or absence of risk factors is not a confirmation for the establishment of cancer or not in an individual, modifying some of the risk factors is crucial in the prevention of breast and other forms of cancer. Tobacco seriously damages our health, and there is every reason to avoid its intake in any form. Both frequent and high consumption of alcohol should be stopped or significantly reduced. Increased consumption of fatty foods and red-meat should be replaced with increase intake of fruits, vegetables and fish. We can control our exposures to ionizing radiation from work place and gadgets such as phones and laptops.
Frequent exercise or moderate physical activities are essential. Preventing other diseases, especially viral infections are crucial to cancer prevention.

\section{Acknowledgements}

None.

\section{Conflict of interest}

The author declares no conflict of interest.

\section{References}

1. Parkin DM. Global Cancer statistics in the year 2000. Lancet Oncol. 2001;2(9):533-543.

2. Boyle NH, Levine DF. Objective and subjective breast cancer risks. Cancer Nurs. 2008;35(8):523-525.

3. Ferlay J, Shin HR, Bray F, et al. Estimates of worldwide burden of cancer in 2008. Int J Cancer. 2008;127(12):2893-2917.

4. Gibbs DF. Cell biology and Cancer. India: Oxford University press; 2003. p. 76-78.

5. Medical news today. Breast cancer; 2013.

6. Bray F, Møller B. Predicting the future burden of cancer. Nat Rev cancer. 2006;6(1):63-74.

7. Abdulkarem F. Epidemiology and incidence of common cancers in Nigeria. Negiria: University of Lagos press; 2017.

8. Globocan. Cancer incidence and mortality worldwide; 2012.

9. IARC International agency for research on cancer. Handbook on breast cancer. France: Springer; 2011. p. 44-45.

10. Cancer research. Cancer, causes and symptoms; 2013.

11. American cancer society. Cancer in Africa. Atlanta: G.A American cancer society; 2011.

12. Olsen M. Cancer in Sub-sahara Africa. The need for new paradigms in global health. England: Boston University Press; 2015.

13. Perou CM, Sorlie T, Eisen MB, et al. Molecular portraits of breast tumours. Nature. 2000;406(96797):747-752.

14. Curtis C, Shah SP, Chin SF, et al. The genomic and transcriptomic architecture of 2,000 breast tumours reveals novel subgroups. Nature. 2012;486(7403):346-352.

15. Morgan JG. Position Paper of the American council on science and health on the risk factors of breast cancer. The Breast Journal. 2013;4(3):177197.

16. Ly M, Antoine M, Andre F, et al. Breast cancer in Sub-Saharan African Women. Bull Cancer. 2011;98 (7):798-805.

17. Elgailli EM, Abuidriss D, Rahman M, et al. Breast cancer burden in central Sudan. Int J Womens Health. 2010;9(2):77-82.

18. American cancer society. Global Cancer Facts \& Figures. 2nd ed. Georgia: American Cancer Society; 2011. p. 55-56.

19. Harris J, Lippman M, Veronesi U. Breast cancer (part 3). $N$ Engl J Med. 1992;327(7):319-322.

20. Greenlee RT, Hill-Harman MD, Murry TT. Cancer statictics. Cancer J Clin. 2001;15(6):402-406.

21. Cancer statistics worldwide. London cancer research report (No.104) UK: Springer; 2005. p. 45-46. 
22. Althuis MD, Dozier JM, Anderson WF, et al. Global trends in breast cancer and mortality. Int J Epidemiol. 2005;34(2):405-412.

23. Garcia M. Global Cancer Facts \& Figures. 3rd ed. American Cancer Society, Georgia: 2009. 67 p.

24. Cancer factsheet. Geneva: WHO; 2011.

25. Baba AJ and Hincal E. Cancer incidence in nigeria and border countries. Malys J Med biol Re. 2016;3(1):10-11.

26. International agency for research on cancer. Monographs on the evaluation of carcinogenic risks to humans. Ionizing radiation, Part 1. Vol 75, France: International Agency for Research on Cancer Press 2000 .

27. Robbins SL, Cotran RS, Kumar V. Pocket companion: pathologic basis of disease. 2nd ed. Philadelphia, USA; 2001.

28. International agency for research on cancer. IARC handbooks of cancer prevention. Weight control and physical activity. Vol 6, France: IARC Press; 2002

29. Malone KE, Daling JR, Thompson JD, et al. BRCA 1 mutation and breast cancer in the general population. Analysis in women before age 35 years and in women before age 45 years with first-degree family history. JAMA. 1998;279(12):922-929.

30. Haber D. Prophylactic Ocphorectomy to reduce the risk of Ovarian and the risk of ovarian and breast cancer in carriers of BRC mutations. $N E n g l$ J Med. 2002;346(40):1660-1661.
31. Greene MH. Genetics of breast cancer. Mayo Clin Proc. 2002;7(2):54 65.

32. Cancer research. Healthy living; 2013.

33. Byrne C, Brinton LA, Haile RW, et al. Heterogeneity of the effect of family history on breast cancer risk. Epidemiology. 1991;2(4):276-284.

34. Carey LA. Through a glass darkly: advances in understanding breast cancer biology, 2000-2010. Clin Breast Cancer. 2010;10(3):188-195.

35. Shaukat U, Ismail M, Mehmood N. Epidemiology, major risk factors and genetic predisposition for breast cancer in the Pakistani population. Asian Pac J Cancer Prev. 2013;14(10):5625-5629.

36. Ruder EH, Dorgan JF, Kranz S, et al. Examining breast cancer growth and lifestyle risk factors: Early Life, Childhood, and Adolescence. Clinical Breast Cancer. 2008;8(4):334-342.

37. Union for international cancer control. Cancer explained. Geneva, Switzerland: UICC press; 2013

38. Briton L, Bornstein L, Colditzc. Workshop on physical activity and breast cancer. Cancer. 1998;83(6):595-600.

39. Costanza ME. Epidemiology and risk factors for breast cancer. Update. $2001 ; 9(2): 4-8$ 